\title{
Geometrical approach in atomic physics: Atoms of hydrogen and helium
}

\author{
Oleg Olkhov \\ Institute of Chemical Physics, Moscow, Russia
}

\section{Email address:}

oleg.olkhov@rambler.ru

\section{To cite this article:}

Oleg Olkhov. Geometrical Approach in Atomic Physics: Atoms of Hydrogen and Helium. American Journal of Physics and Applications. Vol. 2, No. 5, 2014, pp. 108-112. doi: 10.11648/j.ajpa.20140205.12

\begin{abstract}
The hypothesis was earlier suggested by the author where all micro-objects are considered as specific distortions of the physical space-time pseudo-Euclidean geometry, namely, as closed topological 4-manifolds. The foundation of the hypothesis is a geometrical interpretation of the basic equation of quantum mechanics for classical (not quantized) wave fields -- the Dirac equation for free particle. Such hypothesis does not contradict to any physical laws and experimental facts and gives firstly an opportunity to explain qualitatively within classical notions (geometrical) the so called "paradoxical" properties of quantum particles such as wave-corpuscular duality, appearance of probabilities in the quantum mechanics formalism, spin, EPR-paradox.To demonstrate prospects for suggested geometrical approach the author early attempted to find new dynamic equations other than known quantum-mechanical ones for atomic spectra calculations. In this work above investigation is being continued on a more rigorous basis, representing a new geometrical interpretation of the equation for hydrogen atoms. Results of calculations of ionization potentials for helium atom are in agreement with experimental data.
\end{abstract}

Keywords: Geometrical Interpretation, Quantum Mechanics, Atomic Spectra, Helium Spectrum

\section{Introduction}

The hypothesis was earlier suggested by the author where all micro-objects are considered as specific distortions of the physical space-time pseudo-Euclidean geometry, namely as closed topological 4-manifolds. For an observer in usual 3 -space such objects look as moving topological defects of this space. The foundation of the hypothesis is a topological interpretation of basic concepts of quantum mechanics mathematical apparatus. Contrary to traditional Copenhagen interpretation, this interpretation gives an opportunity to describe quantum objects between measurement acts (with the help of geometrical notions). Such hypothesis does not contradict to any physical laws and experimental facts and gives firstly an opportunity to explain qualitatively within classical notions (geometrical) the so called "paradoxical" properties of quantum particles such as wave-corpuscular duality, appearance of probabilities in the quantum mechanics formalism, spin, EPR-paradox.

To become a physical theory any hypothesis, even internally uncontroversial, have to suggest (along with qualitative arguments) a new quantitative mathematical apparatus, having advantages over the existing one. To demonstrate prospects for suggested geometrical approach the author attempted in a resent paper to find new dynamic equations other than known quantum-mechanical ones [1]. As a topical problem we selected, as a first step, the geometrical interpretation of known Dirac's equation for hydrogen atom and then we found new equations for spectrum calculations for simplest many electron atom--helium atom. These equations can be considered as the self-consistent field theory that differs from the known Hartree-Fock theory. In this work above investigation is being continued on a more rigorous basis, representing a new geometrical interpretation of the relativistic Dirac equation for hydrogen atoms. Results of calculations of ionization potentials are in agreement with experimental data.

The plan of the paper: in Section 2 we present the main points of suggested hypothesis, in Section 3 we present geometrical interpretation of known relativistic Dirac's equation for hydrogen atom, in Section 4 we present derivation of new equations for helium atom and use them for helium spectra calculations. 


\section{Foundations of the Hypothesis}

Let us remind at first main points of the suggested geometrical interpretation of quantum mechanics [2,3]. The starting moment was the topological interpretation of Dirac's relativistic equation for free particle with spin $1 / 2$. Symmetric form of this equation is [4]

$$
\hat{\mathrm{p}}_{\mu} \gamma_{\mathrm{ik}}^{\mu} \psi_{\mathrm{k}}=\mathrm{m} \psi_{\mathrm{i}}
$$

$\mathrm{m}$ is the mass of the particle, $\hat{\mathrm{p}}^{\mu}=\mathrm{i} \partial^{\mu}=(\mathrm{i} \partial / \partial \mathrm{t},-\mathrm{i} \nabla)$, $\gamma^{\mu}(\mu=0,1,2,3)$ are four-row Dirac's matrices, $\psi_{\mathrm{i}}(\mathrm{x})$ Dirac's byspinor, $i=1,2,3,4$. We use in (1) relativistic units where $\hbar=\mathrm{c}=1$. Here and later we use 4-metrics with signature $(+---)$. With such units the elementary charge square is $e^{2}=1 / 137$. Solution of ( 1 ) for free particle's states with definite values of 4-momentum $\mathrm{p}_{\mu}$ has the form of plane wave

$$
\psi_{\mathrm{p}}=\mathrm{u}_{\mathrm{p}} \exp \left(-2 \pi \mathrm{ix}{ }^{\mu} \lambda_{\mu}^{-1}\right)
$$

where $u_{p}--$ definitely normalized byspinor and

$$
\lambda_{1}^{-2}-\lambda_{2}^{-2}-\lambda_{3}^{-2}-\lambda_{4}^{-2}=\lambda_{\mathrm{m}}^{-2}, \lambda_{\mu}=2 \pi \mathrm{p}_{\mu}^{-1}, \lambda_{\mathrm{m}}=2 \pi \mathrm{m}^{-1}
$$

Within traditional interpretation (1) describes only possible results of measurements over quantum object, namely, possible values of its 4-momentum. But this equation does not include no another information about the object before or between measurements (for example, what is it within classical notions). It is supposed in our hypothesis that (1) includes such information. The starting point of the hypothesis is the fact that above solution of (1) can be interpreted as a basic vector of a representation for the infinity translation group, including all translation of the form

$$
\mathrm{s}_{\mathrm{n}}=\mathrm{n}_{0} \lambda_{0}-\mathrm{n}_{1} \lambda_{1}-\mathrm{n}_{2} \lambda_{2}-\mathrm{n}_{3} \lambda_{3} .
$$

Here $\lambda_{0}, \lambda_{1}, \lambda_{2}, \lambda_{3}$ - four basic orthogonal vectors into four-dimensional pseudo-Euclidean space, $\mathrm{n}_{0}, \mathrm{n}_{1}, \mathrm{n}_{2}, \mathrm{n}_{3}$ integers. The physical pseudo-Euclidean space-time does not have such symmetry (corresponding to a symmetry of infinite crystal), and our main suggestion is that above group works into the definite auxiliary space -- the universal covering space of some closed 4-manifold described by (1). Such spaces are used in topology for description of closed manifolds because discrete groups, working into such spaces, isomorphic to so called manifold's fundamental groups. Elements of this group are different classes of closed paths that starts and finishes at same point (Poincare group $\pi_{1}[4,5]$ ). In particular, an infinite translation group operating in one-dimensional Euclidean space isomorphic to a fundamental group of the one-dimensional closed manifold homeomorphic to a circle. An infinite translation group operating in two-dimensional Euclidean space isomorphic to a fundamental group of the two-dimensional closed manifold homeomorphic to a torus $[5,6,7]$. In addition, the wave function (3) is a byspinor -tensor realizing two-sign representation of a rotation group and therefore can be considered as a description of nonorientable geometric objects [8,9].

Above consideration leads to a hypothesis that (1) can be considered as a description of a closed manifold (by coordinates of its universal covering space), namely, as a description of the closed nonorientable topological space-time 4-manifold, where spin $1 / 2$ corresponds to index of the two-sign rotation group and relation (3) is a metric restriction on possible values of closed paths $\lambda_{\mu}$ in the manifold. It can be shown that due to pseudo-Euclidean metrics of the physical space-time such four-dimensional object represents moving topological defect of three-dimensional space and that this defect has wave-corpuscular and stochastic properties. And this gives an opportunity to identify such geometrical object with the quantum object, described by (1) $[2,3]$

\section{Dirac Equation for Hydrogen Atom}

We start application of the hypothesis to atomic physics (to atomic spectra theory) with the simplest case---with the geometrical interpretation of the Dirac relativistic equation for hydrogen atom [4]

$$
\left(\hat{\mathrm{p}}_{\mu}-\mathrm{A}_{\mu}\right) \gamma_{\mathrm{ik}}^{\mu} \psi_{\mathrm{k}}=\mathrm{m} \psi_{\mathrm{i}},
$$

Where $\mathrm{A}_{\mu}{ }^{--}$nuclear 4-potential. It is supposed within traditional interpretation that (5) describes (as (1) for free particle) only possible results of measurements with atoms but says nothing about (does not explain) what the atom is before and between measurements. In particular, it does not follow from (5) that hydrogen atom contains inside any point-like particle (electron). Our topological interpretation of (5) supposes that this equation describe not only possible results of measurements but also describes specific distortion of space-time Euclidean geometry (existing between measurements) namely the closed nonorientable manifold. Then function $\psi_{\mathrm{i}}(\mathrm{x})$ appears to be a basic vector for representation of a group of symmetry of this object (radial and angle components of wave function realize representation of corresponding subgroups). If we express all notions in (5) through notions with dimensionality of length we obtain instead (5)

$$
\begin{gathered}
\left(\mathrm{i} \frac{\partial}{\partial \mathrm{x}_{\mu}}+\Theta_{\mu}\right) \gamma_{\mathrm{mk}}^{\mu} \Psi_{\mathrm{k}}\left(\mathrm{x}_{\mu}\right)=\frac{1}{\mathrm{~L}_{\mathrm{m}}} \Psi\left(\mathrm{x}_{\mu}\right) \\
\mathrm{x}_{\mu}=\left(\mathrm{t}_{\mathrm{a}}, \mathrm{x}_{\mathrm{a}}, \mathrm{y}_{\mathrm{a}}, \mathrm{z}_{\mathrm{a}}\right)
\end{gathered}
$$

where $\mathrm{L}_{\mathrm{m}}=\hbar / \mathrm{mc}, \Theta_{0}=\alpha / \mathrm{r}, \quad \alpha=1 / 137=\mathrm{e}^{2} / \hbar \mathrm{c}$. According to our geometrical interpretation, coordinates $x_{\mu}$ in (6) are not coordinates of the physical space-time and equation (6) itself does not connect any events in this space. We suppose that (6) and (1) are written through coordinates in auxiliary space -through coordinates $t_{a}, x_{a}, y_{a}, z_{a}$ in universal covering space 
of above manifold.

All physical notions in (6) are expressed through variables with dimensionality of length so Planck constant $\hbar$ and light velocity $\mathrm{c}$ are happens to be simply coefficients of translation from conventional variables to "geometrical" ones. For example, energy can be expressed through "inverse" centimeters -- units that are often used in atomic physics.

For above topological interpretation of equation (6) will be possible it is necessary to show that this equation can be considered (like (1)) as an equation written in some auxiliary space playing the role of universal covering space of some closed manifold. To prove this we need to show that 4-potentials in (6) can be considered as connectivities in some non-Euclidean space so that derivatives in (6) can be considered as covariant derivatives in this space. But we need not in fact to prove this in the scope of our work because this was proved earlier within the theory of fiber spaces where it was formally shown (without any connection with suggested hypothesis) that electromagnetic potentials in the Dirac equation (5) can be considered as connectivities in the space of gauge group of this equations (group of phase transformation of wave functions)[10,11].

So we have shown that suggested topological interpretation of quantum objects can be applied not only to equation (1) for free particle but also to equation (5) for particle interacting with the external field. This means that two fundamentally different models for hydrogen atom are possible. They both are described formally by the same equation (with different designations) and therefore they both in the same way corresponds to experimental facts. Within one of them (traditional) the hydrogen atom is considered as a system containing a specific point-like particle -- electron. The wave function in this case is considered as a functions of the physical space-time coordinates. Within another model (geometrical) this atom is considered as a specific stretched microscopic deformation of the physical space-time. The wave function is considered here as a function of coordinates in the definite auxiliary space and these coordinates have nothing in common with coordinates of any point-like particle. The wave function in this case realizes a representation of the object symmetry group.

We see that theoretical calculation of the hydrogen atom spectrum means within both above models solution of the same equations (or (5) or (6)). Therefore, suggested geometrical approach has in this case no practical advantages. But for many-electron atoms geometrical interpretation leads to a principally more simple mathematical formulation of the problem of spectra calculation in compare with the traditional quantum formalism. This follows from the fact that wave functions of many-electron atoms are considered within geometrical approach as tensors realizing a representation for the group of symmetry of closed manifolds describing these atoms. It is naturally to suppose that these tensors will be (as for hydrogen atom in (6)) functions of only one space-time coordinate in corresponding universal covering spaces of above manifolds whereas such functions depend within traditional approach on coordinates of all atomic electrons.
Just this late circumstance makes it impossible to solve Schrodinger's equation with good accuracy for many-electron atoms even with the help of modern computers. Therefore, geometrical approach (being approved) should lead to principally more simple calculations of atomic spectra because equations for functions of only one variable can be usually solved without difficulties by numerical methods. And we will obtain below within geometrical approach the new equations for calculations of the spectrum for simplest many-electron atom -- the helium atom.

\section{Spectrum of the Helium Atom}

The spectrum of helium atoms is defined in nonrelativistic limit by the known Schrodinger equation. In atomic units this equation is (see, for example, [12])

$$
\Delta_{1} \mathrm{u}+\Delta_{2} \mathrm{u}+2\left(\mathrm{E}+2 / \mathrm{r}_{1}+2 / \mathrm{r}_{2}-1 / \mathrm{r}_{12}\right) \mathrm{u}=0
$$

Where $r_{1}$ and $r_{2}$-- distances between nuclear and first and second electrons, $r_{12}--$ distance between electrons $\Delta_{1}=\partial^{2} / \partial x_{1}^{2}+\partial^{2} / \partial y_{1}^{2}+\partial^{2} / \partial z_{1}^{2}--$ Laplacian in the space of first electron, $\mathrm{u}$-- function of six coordinates $\mathrm{x}_{1}, \mathrm{y}_{1}, \mathrm{z}_{1}$ and $\mathrm{x}_{2}, \mathrm{y}_{2}, \mathrm{z}_{2}$.

Equation (7) is not relativistic invariant. It is impossible to write here (to contrast to (5)) the closed equation that takes into account relativistic corrections for helium atom. The reason is the electron-electron Coulomb potential in (7) whose relativistic corrections can be represented only as a series expansion over powers of the fine structure constant [12]. Unlike $(5,6)$, the solution of (7) cannot be expressed in a closed analytical form, and even approximate solutions can be rarely obtained without numerical calculations [12]. Nevertheless theoretical values for the spectrum of helium atom obtained from (7) are in good agreement with experimental data (with accuracy to relativistic corrections).

The helium atom (as a hydrogen atom) is considered within suggested geometrical approach as some localized distortion of the space Euclidean geometry and there is now no necessity for its representation as a system containing two identical point-like particles-electrons being in external field. But for finding out new equations for helium atoms (within geometrical model) it is necessary to take into account two new factors that are absent in equation (6) for hydrogen atoms. Firstly, helium atom has a symmetry with respect to permutation of two identical electrons. Secondly, helium atom has an additional energy due to electron Coulomb interaction. These two factors are direct consequence of the atomic model with two point-like electrons, and both these factors should be presented in mathematical description within geometrical approach that does not consider such particles. To take into account permutation invariance we suppose that the wave function is a basis for representation of the group of permutations of second rank and that the wave function depends within geometrical approach on only one space-time coordinate. This means that we consider a permutation group 
of second rank as a group isomorphic to the group of some symmetric transformations of the geometrical object, representing the helium atom. It is well known that a permutation group can be isomorphic to a group of symmetry transformations of some geometrical object: the classical example is a isomorphism of permutation group of third rank and the group of symmetry transformations of the plane equilateral triangle [13]. Therefore, inclusion of a permutation group in symmetry groups of the geometrical object representing helium atoms means that its description within geometrical approach has to use a tensor, depending (firstly) on one space-time coordinate and (secondly) realizing a representation of the permutation group of second rank.

The simplest such tensor is a 4 -vecor $\mathrm{B}_{\mu}$ that realizes representation of the inversion group isomorphic to a permutation group of second rank [4]. Then initial equations for the vector $\mathrm{B}_{\mu}$ (without taking into account interaction of electrons to one another and with nuclear) should be relativistic invariant relations obtained with the help of operators $\hat{\mathrm{p}}^{\mu}$ and containing electron mass.

The simplest such relation relations are (in relativistic units)

$$
\hat{\mathrm{p}}^{2} \mathrm{~B}_{\mu}=\mathrm{m}^{2} \mathrm{~B}_{\mu}
$$

where $\hat{\mathrm{p}}^{2}=\hat{\mathrm{p}}^{v} \hat{\mathrm{p}}_{v}, \mathrm{~m}--$ mass of an electron.

The next step is inclusion in (8) interactions of electrons to one another and with nuclear. Nuclear potential is introduced in (8) in the same manner as this potential was introduced in (1) for obtaining equation (5) for hydrogen atom, that is, by lengthening of derivatives. So after taking nuclear potential into account equation (8) take the form

$$
\hat{\mathrm{P}}^{2} \mathrm{~B}_{\mu}=\mathrm{m}^{2} \mathrm{~B}_{\mu},
$$

Where $\hat{\mathrm{P}}^{2}=\hat{\mathrm{P}}^{v} \hat{\mathrm{P}}_{v}, \hat{\mathrm{P}}_{v}=\hat{\mathbf{p}}_{v}-\mathbf{A}_{v}, \mathrm{~A}_{v}$-- 4-potential of the nuclear (for example, $A_{0}=-2 / r$ ).

There is no any guidance how to introduce in (8) interaction corresponding to Coulomb electron-electron interaction. We suppose that this interaction can be taken into account by introducing in (9) additional potential $\mathrm{V}_{v}(\mu)$, that is defined, as $A_{v}$, from Maxwell's relativistic equations for the tensor of electromagnetic field $F_{v \alpha}(\mu)[14]$

$$
\partial^{\alpha} F_{v \alpha}(\mu)=j_{v}(\mu), \quad F_{v \alpha}(\mu)=\partial_{v} V_{\alpha}(\mu)-\partial_{\alpha} V_{v}(\mu)
$$

Here $\mu$ in brackets indicates on belonging to corresponding equation in (9). Currents $j_{v}(\mu)$ in the right side part of (10) are defined as conserving currents corresponding to different components of 4-vector from (9). Before to define these currents we suggest that the equation for $\mathrm{B}_{0}$ describes atomic states with spin zero corresponding to para-helium and equations for other components in (9) $B\left(B_{1}, B_{2}, B_{3}\right)$ describe states with spin one corresponding to orto-helium. Using known expressions for currents of wave fields with spin one or zero we obtain for scalar and vector 4-currents [4]

$$
\begin{gathered}
j(0)=j_{v}^{c}=2 i\left(B_{0}^{*} \frac{\partial B_{0}}{\partial x^{v}}-B_{0} \frac{\partial B_{0}^{*}}{\partial x^{v}}\right), \\
j(1)=j(2)=j(3)=j_{v}^{B}=2 i\left(B^{v k *} B_{k}-B^{v k} B_{k}^{*}\right),(k=1,2,3) \\
i B_{\alpha \beta}=\hat{p}_{\alpha} B_{\beta}-\hat{p}_{\beta} B_{\alpha},
\end{gathered}
$$

Where coefficient 2 is an electron's doubled charge. Above currents do not depend on time because their expressions contain binary combinations of complex conjugate functions and $V_{v}(\mu)$ satisfied the Poisson equation (this follows from (10))[14]

$$
\Delta V_{v}(\mu)=j_{v}(\mu), \quad V_{v}(\mu)=\int \frac{j_{v}(\mu) d^{3} r_{1}}{\left|r-r_{1}\right|}
$$

Finally, the required system of relativistic invariant equations for calculation of the energy levels of helium are

$$
\begin{gathered}
\hat{\mathrm{P}}^{2}(\mu) \mathrm{B}_{\mu}=\mathrm{m}^{2} \mathrm{~B}_{\mu}, \\
\hat{\mathrm{P}}^{2}(\mu)=\hat{\mathrm{P}}^{v}(\mu) \hat{\mathrm{P}}_{v}(\mu), \hat{\mathrm{P}}_{v}(\mu)=\hat{\mathrm{P}}_{v}-\mathrm{A}_{v}-\mathrm{V}_{v}(\mu), \\
\mathrm{V}_{v}(\mu)=\int \frac{\mathrm{j}_{v}(\mu) \mathrm{d}^{3} \mathbf{r}_{1}}{\left|\mathbf{r}-\mathbf{r}_{1}\right|} .
\end{gathered}
$$

Let us consider above equations in nonrelativistic limit. The quantum object's relativistic energy contains the rest energy $\mathrm{m}$. Therefore we have to exclude this energy for transition to nonrelativstic limit by introducing in (14) functions $B_{\mu}^{\prime}$ instead $B_{\mu}$

$$
B_{\mu}=B_{\mu}^{\prime} \exp [-i(m+E) t]=v_{\mu} \exp (-i m t) .
$$

This expression contains the mass of only one electron (but not two electrons). It means that zero ionization energy $\mathrm{E}=0$ corresponds to excitation in the continuous spectrum of only one electron. In other world our equations describe one-particle excitations with mass $m$ (therefore the initial equation (9) contain a unit mass).

Let us insert now (15) in (14) and take into account that differentiation with respect to time leads to appearance of the large factor $\mathrm{m}$. Then we neglect of terms of the order $\mathrm{E} / \mathrm{m}$. In result we obtain the following equations for calculation of helium spectrum

$$
\left(\frac{1}{2 \mathrm{~m}} \Delta+\frac{2}{\mathrm{r}}-\int \frac{\left|\mathrm{v}_{\mu}\left(\mathrm{r}_{1}\right)\right|^{2} \mathrm{~d}^{3} \mathrm{r}_{1}}{\left|\mathrm{r}-\mathrm{r}_{1}\right|}+\mathrm{E}\right) \mathrm{v}_{\mu}(\mathrm{r})=0 .
$$

These equations are similar to equations of the theory of self-consistent field suggested by Hartree and Fock [12], but they are more simple that makes its solution by numerical methods more easy. Let us consider some solutions of above equations for the case of symmetrical $\mathrm{s}$-states. In this case 
(16) takes the form (in atomic units)

$$
\begin{aligned}
& \left(\frac{d^{2}}{d r^{2}}+\frac{2}{r} \frac{d}{d r}+\frac{4}{r}-2 E-2 \int \frac{\left|v\left(r_{1}\right)\right|^{2} d^{3} r_{1}}{\left|r-r_{1}\right|}\right) v=0 \\
& \left(\frac{d^{2}}{d r^{2}}+\frac{2}{r} \frac{d}{d r}+\frac{4}{r}-\frac{2}{r^{2}}-2 \varepsilon-2 \int \frac{\left|u\left(r_{1}\right)\right|^{2} d^{3} r_{1}}{\left|r-r_{1}\right|}\right) u=0,
\end{aligned}
$$

Where $u^{2}=u_{x}^{2}+u_{y}^{2}+u_{z}^{2}$. Notice that equation (18) for orto-helium differs from equation (17) for para-helium by presence of centrifugal potential that reflects the symmetry of this state corresponding to spin 1 . The numerical solution of (17) with accuracy to second decimal place gives for ionization potential of para-helium $\mathrm{E}=0.92$. The experimental value is $\mathrm{E}_{\mathrm{exp}}=0.91$, and the value from the Hartree-Fock theory is $\mathrm{E}_{\mathrm{HF}}=0.86$ [12]. Numerical solution of (18) gives for ionization potential of orto-helium $\varepsilon=0.19$, while the experimental value is $\varepsilon_{\exp }=0.18$ [12].

\section{Conclusion}

We see that an accuracy of results obtained from the new equations is the same as in Hartree-Fock theory (several percent). This means that new equations (14) cannot be used for calculation of relativistic corrections having the order of $\alpha^{2} \sim 10^{-5}$, where $\alpha$ - the fine structure constant [12]. Notice that relativistic derivation of the equations was required only for correct accounting of the considered symmetries. It should be stressed in conclusion that the main goal of the work is not to improve the accuracy of Schrodinger's equation for helium atom but to find out the possibility for reflection of physical reality within principally new approach in quantum physics. As it was here shown (at least approximately) the suggested geometrical description of the helium atom reflects reality correctly.

\section{Acknowledgements}

It is my pleasure to thank V.L.Bodneva and A.S.Prostnev for their help in numerical calculations.

\section{References}

[1] O. A. Olkhov, "Geometrical approach to the atomic spectra theory. The helium atom," Russian J.of Phys.Chemistry B, vol. 8, pp.30 - 42, February 2014 [Chim.Fis., p.36, vol.33, №2, 2014].

[2] O. A. Olkhov, "Geometrization of Quantum Mechanics," Journal of Rhys.:Conf.Ser. vol. 67, p.012037. 2007.

[3] O. A. Olkhov, "Geometrization of Classical Wave fields," Mellwill, Ney York, AIP Conference Proceedings p. 316, vol.962,2008(Proc.Int.Conf,"Quantum theory: Reconsideration of Foundations, Vaxjo, Sweden, 11-16 June, 2007, 2007); arXiv: 802.2269

[4] L. D. Landau, E. M. Lifshitz, Course of Theoretical Physics. vol.4: V. B. Berestetzki, E. M. Lifshitz, L. P. Pitaevski, Quantum Electrodynamics, Butterworth-Heinemann, 1982.

[5] B. A. Dubrovin, A. T. Fomenko, S. P. Novikov, Modern geometry-Methods and Applications, Part 2: The Geometry and Topology of Manifolds, Springer, 1985.

[6] A. S. Schwartz, Quantum field theory and topology. Grundlehren der Math. Wissen.307, Springer, 1993.

[7] H. S. M. Coxeter, Introduction to geometry. New York, London: John Wiley\&Sons, 1961.

[8] P. K. Rashevski, Riemann geometry and tensor analysis (in Russian). Moscow, Nauka, 1966.

[9] V. A. Zelnorovich, Theory of spinors and its applications (in Russian). Moscow, August-Print, 2001.

[10] Ta-Pei Cheng, Ling-Fong Li, Gauge theory of elementary particle physics. Clarendon Press, Oxford, 1984.

[11] V. Popov, N. Konopleva, Gauge fields. Gordon and Breach Publishing Group, 1982.

[12] H. A. Bethe and E. E. Salpeter, Quantum mechanics of one- and two-electron atoms. Berlin-Gottingen-Heidelberg, Springer-Verlag, 1957.

[13] V. Heine, Group theory in quantum mechanics. London-Oxford-New York-Paris, Pergamon Press, 1960.

[14] L. D. Landau, E. M. Lifshitz, Course of Theoretical Physics, vol.2, L. D. Landau, E. M. Lifshitz, The Classical Theory of Fields, Butterworth-Heinemann, 1975. 